\title{
The effect of the epigenome on the Vitamin D Receptor target genes
}

\author{
K. de Nooijer \\ Bioinformatics-BiGCaT, Maastricht University \\ k.denooijer@student.maastrichtuniversity.nl
}

\section{Abstract}

Background. Humans produce vitamin $D_{3}$ by exposure to sunlight that includes ultraviolet $B$ (UVB) radiation, but also via the intake of nutrients. In the body, the Vitamin $D_{3}$ is converted to its active form, which is called $1,25(\mathrm{OH})_{2} \mathrm{D}_{3}$. This can bind to the Vitamin D Receptor (VDR), which is located on several genes which are called the VDR target genes. Objective. The present study investigated which genes are regulated by the 1 , ${ }_{25}(\mathrm{OH})_{2} \mathrm{D}_{3}$ and checked whether the epigenetic modifications are measured in two cell lines within the ENCODE project. The data of the epigenetic modifications will be linked to the promoter regions of the VDR target genes. In this way the epigenetic control of the VDR target genes could be investigated in more detail. Methods. The human VDR target genes were identified with an extensive literature search. The promoter regions of the VDR target genes were found with Genomatix. Two types of epigenetic modifications, DNA methylation and histone modification, which were determined in two cell lines, IMRgo and $\mathrm{H}_{1} \mathrm{ESC}$, in the ENCODE project were linked to the promoter regions of the VDR target genes. In the network analysis program Cytoscape the VDR target genes and the measured epigenetic modifications were integrated and visualized in an epigenetics-VDR target genes network. Results. In total 185 VDR target genes were found in 25 literature papers. There were 528 promoter regions in the 185 VDR target genes identified. In the $\mathrm{H}_{1}$ ESC were 445 histone modifications linked to the VDR target gene promoters and in the IMRgo were 259 histone modifications linked to the VDR target gene promoters. In the IMR9o were 158 promoter regions with a DNA methylation region and in the $\mathrm{H}_{1} \mathrm{ESC}$ were this 177 promoter regions with a DNA methylation region. In the combined network of DNA methylation and histone modifications were in the IMR9o 99 genes and in the $\mathrm{H}_{1}$ ESC 111 genes selected. Conclusion. The epigenetics-VDR target genes network can be used in further research to understand the role of the epigenetic control of the VDR target genes. 


\section{Keywords}

Human genome, vitamin D3, VDR, VDR target genes, epigenome.

\section{Introduction}

Epigenetic mechanisms play a crucial role in the regulation of gene expression (1). The main mechanisms involve DNA methylation and histone modifications. DNA methylation and histone modifications are identified in the Encyclopedia of DNA Elements (ENCODE), the ENCODE has advanced the understanding of the principles of the genome, epigenome and chromatin organization. The goal of the ENCODE is to identify the functional elements in the human genome, which will be done with different assays and methods (2). The external environment plays a role in the regulation of the epigenome, different factors can switch genes on or off and determine which proteins are transcribed (3). One of these factors is Vitamin D, which interacts with the epigenome (1). The intake of Vitamin D3 intake is unique because it is not only obtained by the exposure of sunlight but also via the intake of nutrients (4). In the body, the Vitamin $\mathrm{D}_{3}$ is converted in the liver and kidneys to the active form of Vitamin $\mathrm{D}_{3}, 1,25(\mathrm{OH})_{2} \mathrm{D}_{3}$. The $1,25(\mathrm{OH})_{2} \mathrm{D}_{3}$ can bind to the Vitamin $\mathrm{D}$ Receptor (VDR) (4). The 1, 25(OH)2D3 acts as a ligand agonist for the VDR, and the liganded VDR activates target gene expression at the transcriptional level (5). Genes influenced by the $1,25(\mathrm{OH})_{2} \mathrm{D} 3$ are called the VDR target genes (4). Between 1,600 and 2,800 genomic VDR binding sites have been determined upstream or downstream of the transcription start site of the VDR target genes, however, only a minor set of these VDR binding sites has been confirmed in multiple analyses (6). The expression of the VDR at baseline is measured in various tissues, for example the colon, lung and bone marrow (7).

This data leads to the following research questions: What are the vitamin D receptor target genes found in the human genome and in which tissue and with which method are they measured? How can we link epigenetic modifications to the genes? How can the epigenetic control of the VDR target genes be visualized?

\section{Material and methods}

The VDR target genes in the human genome were identified by the use of different literature sources. The promoter regions of the VDR target genes were found, this data was used to identify the histone modifications and DNA methylation in the promoter regions of the VDR target genes. The VDR target genes and the epigenetic modifications were integrated and visualized. 


\section{Identify vitamin $\mathrm{D}$ target genes}

The identification of the VDR target genes was started with searching on the internet for relevant articles about Vitamin $D_{3}$. This was done with the use of PubMed, with the search term "VDR target gene". The analysis resulted in a list of papers related to the VDR target gene, which then were read if they provided information about the human genome. This was also done with the search terms, "VDR" and "gene regulation vitamin D". The genes mentioned in the literature were categorized in classical VDR target genes and VDR targets in cell cycle regulation.

\section{Promoter region of the VDR target genes in Genomatix}

The software suite Genomatix was used to find the information about the promoter region of the genes. In Genomatix was the module Gene2Promoter, which gave the access to promoter regions of all the genes for retrieving and analyzing them. In Gene2Promoter were the promoter regions annotated and validated according to scientific standards, including Genomatix technology, for example oligo-capping, Promoterlnspector and comparative genomics (8). The human genome was used, with the NCBI build 37 and ElDorado 12-2013 version, the ElDorado is the Genomatix genome annotation. The gene name was filled in and all the promoters of this gene were extracted directly from the ElDorado 12-2013.

\section{Histone modifications and DNA methylation}

The promoter regions which were found with the software suite Genomatix were used for finding the location of the histone modifications in the VDR target genes. The ENCODE data was collected from two cell types, the H1ESC and IMRgo cell lines. The histone modifications were identified with ChIP-sequencing. This gave as a result the information about the histone modifications of the VDR target genes in base pairs, in which cell type and the description about the histone modification. The DNA methylation data in the IMRgo and $\mathrm{H}_{1} \mathrm{ESC}$ was found in the ENCODE project, which was identified by the use of Reduced Representation Bisulfite Sequencing (RRBS). This data was retrieved with an $R$ script, and the R script was linked to the file with the promoter regions of the VDR target genes with the program Java.

\section{Integration and visualization in Cytoscape}

In the network analysis program Cytoscape was the information of the different histone modifications and DNA methylation visualized for both cell lines, IMRgo and $\mathrm{H}_{1} \mathrm{ESC}$. The data of the DNA methylation and histone modifications in the IMRgo and H1ESC was 
integrated, this gave a network of both the DNA methylation and histone modifications linked to the promoter regions of the VDR target genes in the IMRgo and $\mathrm{H}_{1}$ ESC cell lines.

\section{Results}

In total were 185 VDR target genes found in the literature, these genes were measured with different methods. The promoter regions of these genes were identified by the program Genomatix which gave all the promoter regions in base pairs of the VDR target genes, in total were 528 unique promoter regions found. There was looked to the DNA methylation and histone modifications in two cell lines, IMRgo and $\mathrm{H}_{1} \mathrm{ESC}$, within the ENCODE project. This data was linked to the promoter regions of the VDR target genes and thereafter integrated and visualized in Cytoscape.

\section{Vitamin D target genes}

The VDR target genes were found in literature from various papers. In total were 185 VDR target genes found, there were 167 classical VDR target genes and 18 VDR targets in cell cycle regulation found. These genes were measured with different methods and found in different human tissues. The VDR target genes were found in 25 literature sources, with the publication date varied from 2002 to 2014.

\section{Genomatix}

In the 185 VDR target genes were all the promoters directly extracted from the ElDorado 12-2013 with the module Gene2Promoter. Some genes had only one promoter region but there were also genes which had more promoter regions. In total were 528 unique promoter regions found in the 185 VDR target genes, mostly with the length of 601 base pairs.

\section{Histone modifications and DNA methylation in IMR90 and H1ESC}

In the IMRgo and $\mathrm{H}_{1} E S C$ were 528 promoter regions found in the VDR target genes. In the $\mathrm{H}_{1} \mathrm{ESC}, 445$ histone modifications were linked to the VDR target gene promoters. In the IMRgo cells, 259 histone modifications were linked to the VDR target gene promoters. There were in both cell types different types of histone modifications found, both acetylation and methylation were found in different regions. In the IMR9o were 158 promoter regions where DNA methylation regions were found with a maximal DNA methylation between the $\mathrm{O}$ and 100 percent. In the $\mathrm{H}_{1} E S C$ were these 177 promoter regions.

\section{Integration and visualization of DNA methylation and histone modification}

The data of DNA methylation and histone modifications in the promoter regions of the VDR target genes was integrated and visualized in Cytoscape. In the IMRgo and H1ESC 
network were some genes with only a DNA methylation or histone modification, but also some genes had both DNA methylation and histone modifications. For example gene TNFAIP3 $_{3}$ in the IMRgo network, this gene was linked with a grey edge to the $\mathrm{H}_{3} \mathrm{~K}_{2} 7 \mathrm{me}_{3}$ and linked to a 100 percent maximal DNA methylation (figure 1). Also gene $\mathrm{CYP}_{3} \mathrm{~A}_{4}$ in the $\mathrm{H}_{1} \mathrm{ESC}$ network, which was linked with a grey edge to the $\mathrm{H}_{3} \mathrm{~K}_{3} 6 \mathrm{me}_{3}$ (figure 2). In both networks was shown that one histone modification caused repression, $\mathrm{H}_{3} \mathrm{~K}_{2} 7 \mathrm{me}$, some of the genes linked to this modification were also linked to DNA methylations with varied maximal DNA methylation percentages (figure 1,2).

\section{Discussion/Conclusion}

In total were 185 VDR target genes in the human genome found in the literature. Divided into 167 Classical VDR target genes and 18 VDR target genes who play a role the in cell cycle regulation. The papers used in this study were published between the years 2002 and 2014, these papers included large studies that investigated VDR target genes. Although most studies were included, it could be possible that a few studies were missed based on the search queries which were used in PubMed. Another aspect about this searching method was that in some articles it was not clear in which genome the VDR target genes were found. Therefore it was possible that some genes mentioned in the papers were identified as VDR target gene in another genome than the human genome. On the other hand, most of the VDR target genes were found in different literature sources by different authors and measured by different methods, and were therefore double checked if they existed in the human genome. This was also checked when there was searched for the Entrez ID of the genes in the HomoloGene, when the gene did not exist in the human genome, the gene was deleted from the list of VDR target genes. Even when some genes where not found due to studies which were missed based on the search queries, the quality of the VDR target genes list was still high.

In two cell lines was looked for histone modifications in the promoter regions. The cell lines $\mathrm{H}_{1}$ ESC and IMRgo were used, there was chosen for these cell lines because the expression of the VDR at baseline was measured in these tissues. In total were 16 types of histone modifications found in the IMRgo, the histone modifications $\mathrm{H}_{3} \mathrm{~K}_{2} 7 \mathrm{me}_{3}$ and $\mathrm{H}_{3} \mathrm{~K}_{3} 6 \mathrm{me}_{3}$ were linked to most of the VDR target genes. One histone modification caused repression of the genes, this was $\mathrm{H}_{3} \mathrm{~K}_{2} 7 \mathrm{me} 3$. This is because the $\mathrm{H}_{3} \mathrm{~K}_{2} 7 \mathrm{me} 3$ was strongly associated with the inactivation of gene promoters in both cell types, this histone modification was caused by the enzyme methyltransferase $\mathrm{EZH}_{2}$ (9). The $\mathrm{EZH} 2$ is part of the $\mathrm{PRC} 2$, which is responsible for the repression of many genes (10). The other histone modifications linked to the promoter regions of the VDR target genes in IMRgo and $\mathrm{H}_{1}$ ESC caused activation of 
the gene promoter (11). For example the CALB1 gene in the IMR9o, this was linked to the $\mathrm{H}_{3} \mathrm{~K}_{3} 6 \mathrm{me} 3$ which caused activation of the gene promoter. The protein encoded for the CALB1 was a calcium-binding protein which included calmodulin and troponin C, where the RNA expression was measured in the lung with Microarray and RNA sequencing (12).

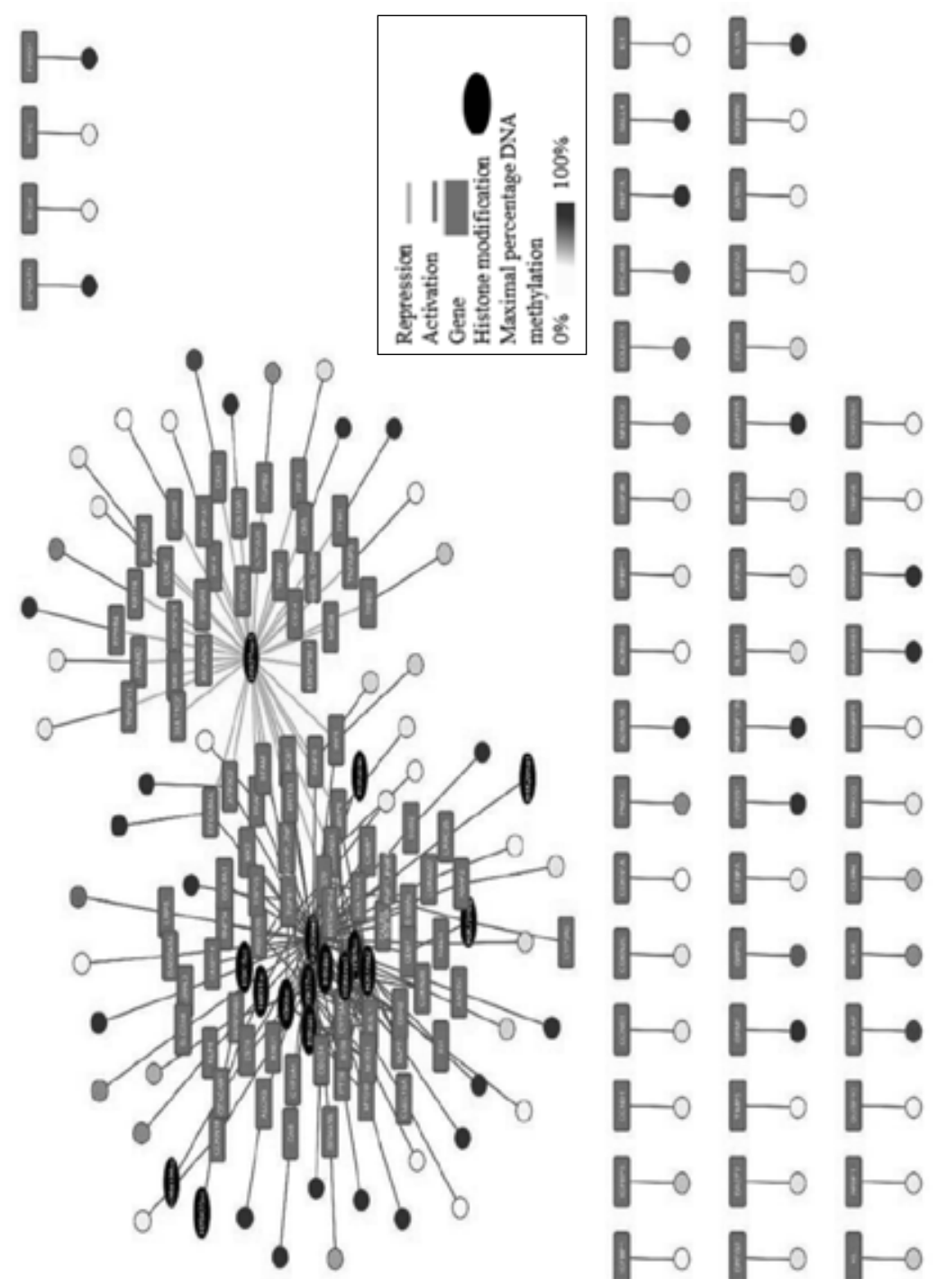

Figure 1. Visualization of DNA methylation and histone modifications in IMRgo. 

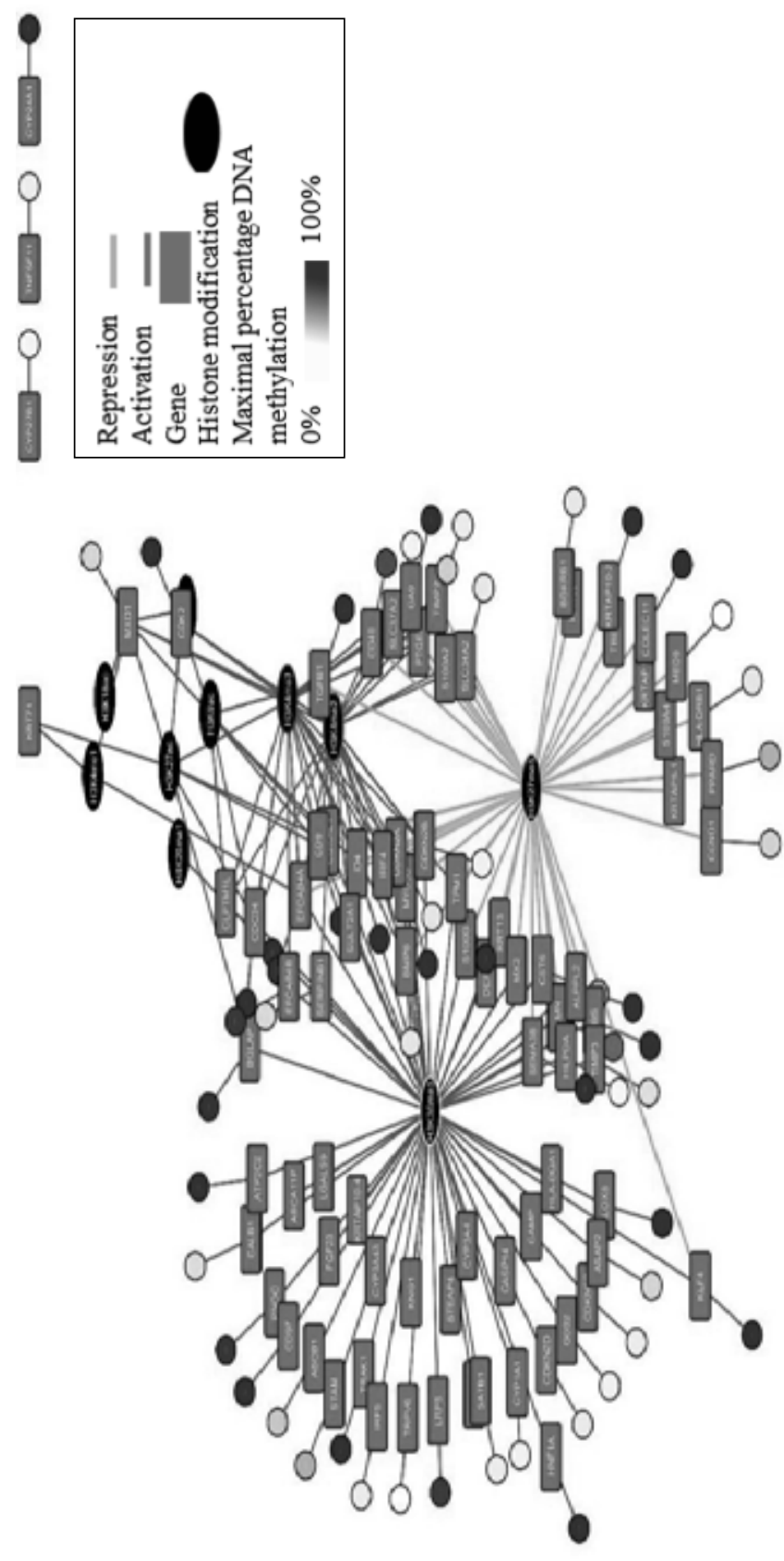

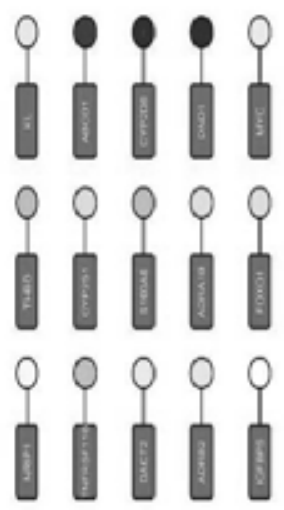

iibil
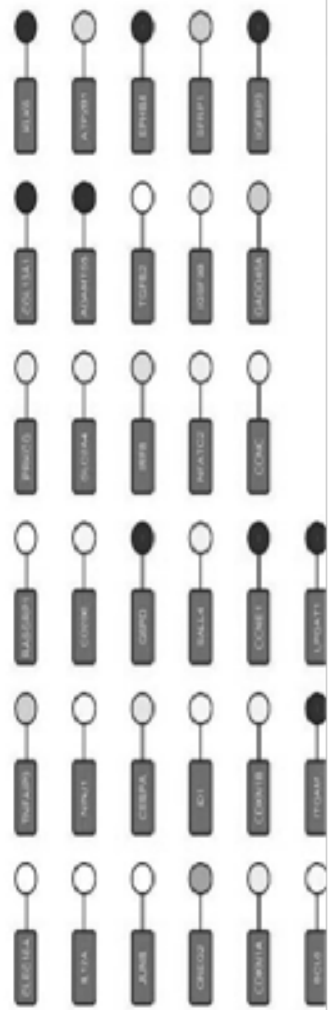

Figure 2. Visualization of DNA methylation and histone modifications in $\mathrm{H}_{1} \mathrm{ESC}$. 
In both cell types were genes found with one promoter region with DNA methylation, but there were also genes with more promoter regions with DNA methylations. The methylation of a cytosine base caused that the promoter region will be blocked for transcription factors and therefore will be repressed (13). There were genes found in the cell lines which had the same maximal DNA methylation percentage, for example CCNC, which was in both cell types surrounded by three promoter regions which all had a maximal percentage of o percent. The CCNC gene encodes for a protein which interacts with cyclin-dependent kinase 8 , which co-activates the phosphorylation of the carboxyterminal domain of nearly all the RNA polymerase 2 dependent genes (12). In both the $\mathrm{H}_{1}$ ESC and IMRgo was the CCNC gene activated, this was because in both cell lines the DNA was copied into messenger RNA (mRNA) by the RNA polymerase 2, which was encoded by the CCNC gene (12).

In the combined network of DNA methylation and histone modification were the promoter regions of the genes selected with the highest maximal DNA methylation percentage. There was shown that in both cell lines some genes were linked to both a promoter region with a DNA methylation region and histone modifications, for example COLEC11 in the $\mathrm{H}_{1} \mathrm{ESC}$, this was linked with $\mathrm{H}_{3} \mathrm{~K}_{2} 7 \mathrm{me} 3$ and to a promoter region with a maximal DNA methylation of 100 percent (figure 2).

In conclusion, the information found in this study can be used in further research to understand the role of the epigenetic control of the VDR target genes.

\section{Role of the student}

In this project I started with the identification of the VDR target genes with an extensive literature search. Secondly, the promoter region in the VDR target genes was identified. Thirdly, two types of epigenetic modifications, DNA methylation and histone modification, which are identified in the ENCODE project were linked to the promoter regions of the VDR target genes. Finally the results were integrated and visualized in a network.

\section{References}

1. Fetahu IS, Hobaus J, Kallay E. Vitamin D and the epigenome. Front Physiol. 2014;5:164.

2. Siggens L, Ekwall K. Epigenetics, chromatin and genome organization: recent advances from the ENCODE project.

3. J Intern Med. 2014;276(3):201-14.

4. Simmons D. Epigenetic Influences and Disease. Nature Education. 2008;1(1):6. 
5. Bendik I, Friedel A, Roos FF, Weber P, Eggersdorfer M. Vitamin D: a critical and essential micronutrient for human health. Front Physiol. 2014;5:248.

6. Deeb KK, Trump DL, Johnson CS. Vitamin D signaling pathways in cancer: potential for anticancer therapeutics. Nat Rev Cancer. 2007;7(9):684-700.

7. Bouillon R, Carmeliet G, Lieben L, Watanabe M, Perino A, Auwerx J, et al. Vitamin D and energy homeostasis: of mice and men. Nat Rev Endocrinol. 2014;10(2):79-87.

8. Expression Atlas [http://www.ebi.ac.uk/gxa/genes/ENSGooooo111424]. 2015.

9. Gene2Promoter [http://www.genomatix.de/online_help/help_eldorado/Gene2Promoter_Intro.html]. 2015.

10. Kuzmichev A, Nishioka K, Erdjument-Bromage H, Tempst P, Reinberg D. Histone methyltransferase activity associated with a human multiprotein complex containing the Enhancer of Zeste protein. Genes Dev. 2002;16(22):2893-905.

11. Rougeulle C, Chaumeil J, Sarma K, Allis CD, Reinberg D, Avner P, et al. Differential histone H3 Lys-9 and Lys27 methylation profiles on the X chromosome. Mol Cell Biol. 2004;24(12):5475-84

12. Peterson C. Histone Modification Table. Cell Signaling Technology. 2007.

13. Science Wlo. GeneCards Human Genome Database. 2015.

14. Philips T. The Role of Methylation in Gene Expression. Nat Educ. 2008;1(1):116. 